\title{
Impact of COVID-19 on Orthopaedic Care and Practice: A Rapid Review
}

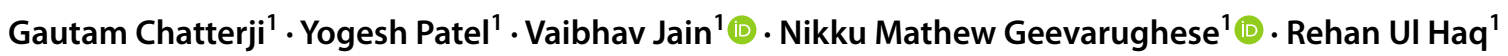

Received: 8 November 2020 / Accepted: 5 January 2021 / Published online: 20 March 2021

(c) Indian Orthopaedics Association 2021

\begin{abstract}
Introduction The COVID-19 pandemic has led to a large body of literature regarding the impact of COVID-19 on orthopaedic care and practice. This rapid review aims to synthesize this published literature to give the orthopaedic fraternity an overview about the best practices that need to be followed during this period.

Methodology A rapid review was conducted following the preferred reporting items for systematic reviews and meta-analyses (PRISMA) statement for rapid reviews on the impact of COVID-19 on orthopaedic care and practice. A Pubmed search was done to identify all literature related to the impact of COVID-19 on orthopaedic care and practice, published between December 2019 and October 2020 using a predefined search strategy. The final review included 375 peer-reviewed articles addressing the objectives.

Results The majority of articles were expert opinions ( $37.1 \%$ ) and narrative reviews (13.1\%). There were $17.3 \%$ retrospective studies and $2.1 \%$ prospective studies with only one randomized control trial and ten systematic reviews. $83.8 \%$ of articles had levels of evidence IV and V, 79.5\% of the articles were published in core-orthopaedic journals. Maximum publications were from the United States of America (31.7\%), followed by India (11.5\%). European countries together contributed to $32.0 \%$ of all publications.

Conclusion COVID-19 has had a significant impact on all aspects of orthopaedic care and practice. The pandemic has affected outpatient clinics, emergency and elective surgery, rehabilitation, resident training, personnel management, use of personal protective equipment, telemedicine and all sub-specialities of orthopaedics. Orthopaedic practice will require the incorporation of new technologies, restructuring of health systems and reorganizing of training programs for optimal patient care. There would also be a need for frequent review of emerging literature to provide evidence-based guidelines to the orthopaedic fraternity which will not only help in mitigation of transmission of disease but also ensure continuity of optimum patient care.
\end{abstract}

Keywords Rapid review · COVID-19 · Orthopaedics · Orthopaedic practice · Elective orthopaedic surgery · Emergency orthopaedic surgery

\section{Introduction}

Nikku Mathew Geevarughese

nikku.mathew@gmail.com

Gautam Chatterji

gautz90@gmail.com

Yogesh Patel

yogeshmonty30@gmail.com

Vaibhav Jain

vaibhavigmc@gmail.com

Rehan Ul Haq

rehan.ortho@aiimsbhopal.edu.in

1 Department of Orthopaedics, All India Institute of Medical Sciences, Bhopal, Madhya Pradesh, India
Ten months into the clutches of Coronavirus Disease-2019 (COVID-19) has shown to affect nearly all walks of life. Initially, the unknown viral spread forced governments to shutdown all non-emergency activities. As knowledge, immunogenicity, transmittance, containment and treatment of the virus increased, these restrictions were pulled off eventually.

It is well understood that orthopaedic surgeons are not on the frontline in managing these patients; however, their expertise has been pertinent when COVID-19 patients have orthopaedic afflictions. Moreover, as orthopaedic surgery is an important element of the overall healthcare ecosystem, 
the effect of COVID-19 pandemic has had an impact on all aspects of orthopaedic care.

Orthopaedic fraternity formulated various guidelines for the management of trauma, emergency and other subspecialties for minimizing exposure to health care workers and patients. A plethora of information has surfaced regarding the management of orthopaedic patients, operation theatres, resident training and virtual clinics among many others. Though numerous directives have been released, they are bound to change as newer research changes the perspective. The rapidly changing guidelines and protocols require quick synthesis and dissemination to guide practice.

Arksey and O'Malley described rapid review as "a type of knowledge synthesis in which components of the systematic review process are simplified or omitted to produce information in a short period of time" [1]. The aim of this rapid review was to synthesize the published literature of the impact of COVID-19 on orthopaedic care and practice to give the orthopaedic fraternity a clear idea about the worldwide overview on outpatient clinics, operating rooms, emergency and elective surgery, personnel management, policy making, personal protective equipment (PPE), resident training, telemedicine and virtual conferencing regarding orthopaedics in the COVID era.

\section{Methodology}

A PubMed search was carried out to identify all literature describing the impact of COVID-19 on orthopaedic care and practice. The study was based on the preferred reporting items for systematic reviews and meta-analyses (PRISMA) statement for rapid review [2,3]. The search terms used were: "orthopaedics" OR "orthopedics" OR "orthopaedic" OR "orthopedic" OR "orthopedic surgery" OR "orthopaedic surgery" OR "orthopedics surgery" OR "orthopaedics surgery" AND "COVID" OR "coronavirus" OR "SARs CoV" in the Title/Abstract (Table 1) and included articles published from 1st December 2019 to 31st October 2020. We identified 390 articles in PubMed®, of which 5 were screened out. Non-English language articles were excluded and articles unrelated to the subject of orthopaedics were deemed ineligible (Fig. 1). The final review included 375 articles, of which the full texts were critically scrutinized over six weeks. We assessed these articles for the type of articles, their levels of evidence, country of origin, month of publication and type of journal. Based on the inputs from all these publications, a generalized overview is provided on outpatient clinics, operating rooms, emergency and elective surgery, personnel management, policy making, PPE, resident training, telemedicine and virtual conferencing and main sub-specialities of Orthopaedics.

\section{Results}

Majority of the articles were found to be expert opinions $(139,37.1 \%)$ and narrative reviews $(49,13.1 \%)$. Retrospective studies made $17.3 \%$ of the publications, while prospective papers were only $2.1 \%$. The study saw only one randomized controlled trial and ten $(2.7 \%)$ systematic reviews (Table 2). Four citations were found to be corrections in earlier published articles. Similarly, $83.8 \%$ of articles had levels of evidence IV and V. Levels I and II publications made up only $3.2 \%$ of this review (Fig. 2). On a positive note, $79.5 \%$ of the articles were published in core-orthopaedic journals, making information readily available to the fraternity. The study noted publications were almost equally released in the months of May, June, August and September, with a salient spike in July (Fig. 3). Authors from the United States of America released the bulk of the articles (31.7\%), followed by India (11.5\%) and the United Kingdom (10.7\%) (Fig. 4). European countries altogether contributed to $32.0 \%$ of all publications.

\section{Policy and Personnel Management}

COVID-19 pandemic forced the global orthopaedic fraternity to change their regular personnel management policies. During the first stage of the crisis, elective surgeries were cancelled and out-patient services were curtailed. In many centres, orthopaedic teams were divided with half strength of the department posted for clinical duties followed by home isolation [4]. The idea being that if a working team gets unprotected exposure to a COVID-19 patient, the reserve team would take charge. All these measures preserved resources (PPE, masks, sanitizers) and prevented undue interaction with patients thereby reducing the exposure to asymptomatic COVID-19 patients. These measures also helped in keeping adequate workforce in reserve to handle the surge in cases. Curtailing elective surgeries

Table 1 Search strategy used to identify relevant citations

Search strategy of PubMed search of articles from December 2019 to October 2020

(orthopaedics[Title/Abstract] OR orthopedics[Title/Abstract] OR orthopaedic[Title/Abstract] OR orthopedic[Title/Abstract] OR orthopedic surgery[Title/Abstract] OR orthopaedic surgery[Title/Abstract] OR orthopedics surgery[Title/Abstract] OR orthopaedics surgery[Title/ Abstract]) AND (covid[Title/Abstract] OR coronavirus[Title/Abstract] OR SARs Cov[Title/Abstract]) 


\section{PRISMA flow chart}

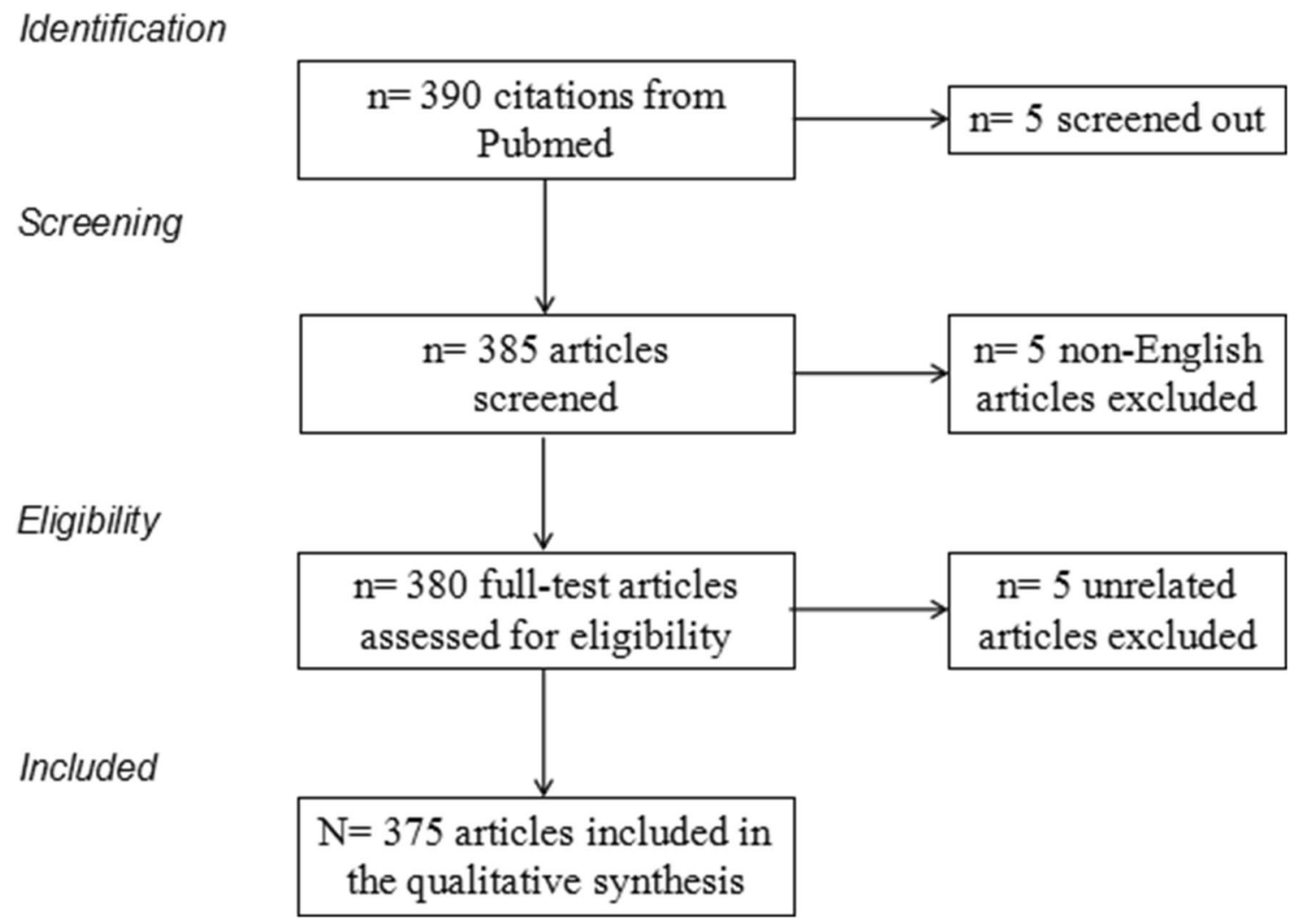

Fig. 1 Preferred reporting items for systematic reviews and meta-analyses (PRISMA) flowchart depicting the flow of selection of articles

Table 2 Distribution pattern of the type of articles

\begin{tabular}{lcc}
\hline Type of article & Number & Percentage \\
\hline Randomised controlled trial & 1 & 0.3 \\
Systematic review & 10 & 2.7 \\
Retrospective study (cohort, com- & 65 & 17.3 \\
$\quad$ parative, others) & & \\
Cross sectional study & 2 & 0.5 \\
Prognostic study & 7 & 1.9 \\
Prospective study (cohort, compara- & 8 & 2.1 \\
$\quad$ tive, others) & 36 & \\
Questionnaire & 23 & 9.6 \\
Letter to Editor & 17 & 6.1 \\
Case series/report & 14 & 4.5 \\
Guidelines & 139 & 3.7 \\
Expert opinion & 49 & 37.1 \\
Narrative review & 4 & 13.1 \\
Corrections & 375 & 100 \\
Total & & \\
\hline
\end{tabular}

enabled general medicine physicians, anaesthetists and other inpatient providers to focus their efforts on addressing the COVID-19 crisis [5]. However, emergency surgeries and services were not stopped in most centres. Orthopaedicians as surgical subspecialists, may not have been on the frontline in the present pandemic, but their contributions have had a considerable impact on the healthcare system during this period [6]. They addressed this nontraumatic healthcare crisis by increasing telehealth capabilities, focusing on consultative care to emergency departments, and helping to direct patients with traumatic injuries toward hospitals that were not overburdened by the management of COVID-19 patients.

Training hardened orthopaedic surgeons due to their versatility and fast learning capabilities, when asked to participate outside of their speciality area in inpatient and intensive care units to look after the pandemic affected patients, were able to do so with basic training and close supervision by their physician counterparts [4]. 
Fig. 2 Distribution pattern of levels of evidence of the articles ( $n=371$, excluding four corrections/erratum)

\section{Levels of Evidence}

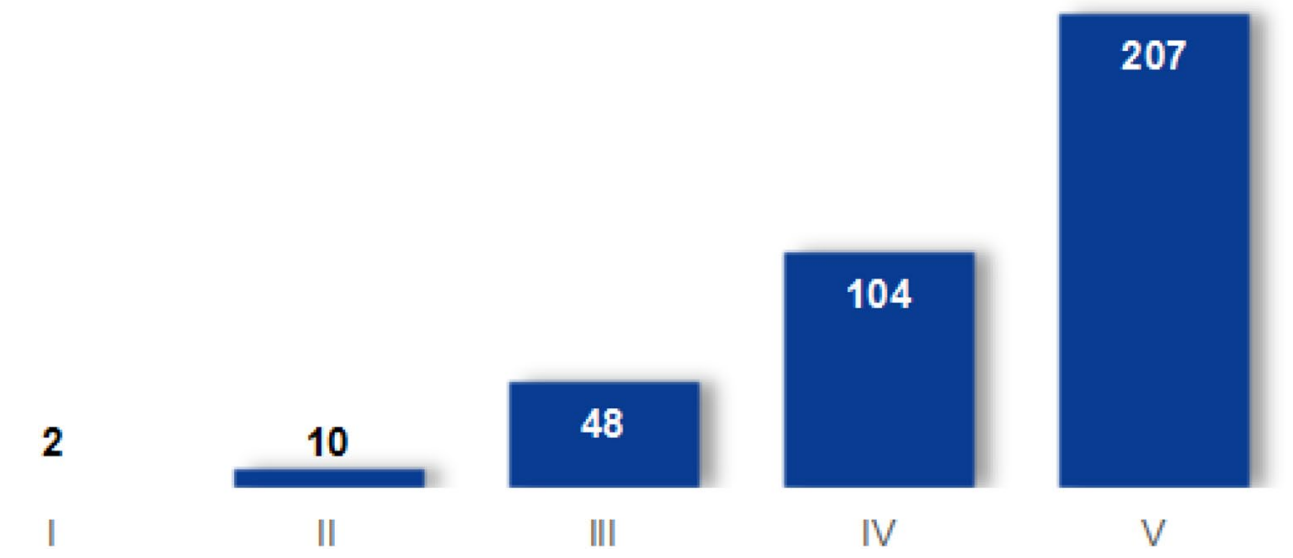

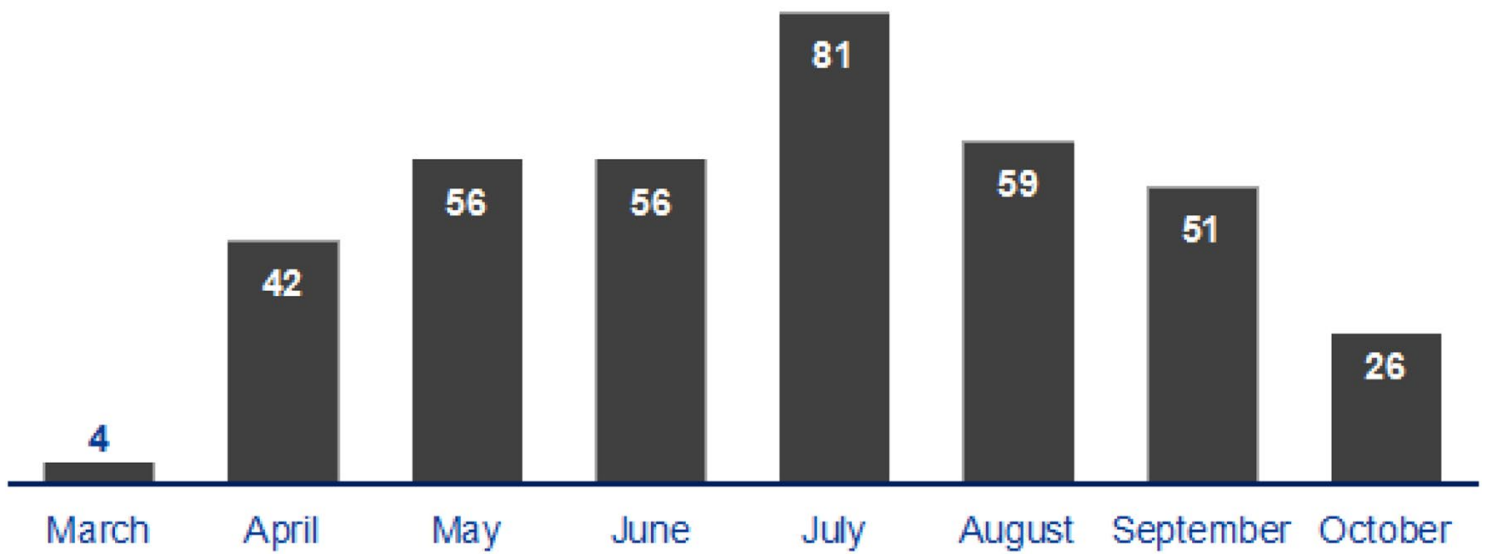

Fig. 3 Distribution pattern of month of publication of the articles $(n=375)$

\section{Personal Protective Equipment}

The Centre for Disease Control (CDC) and World Health Organization (WHO) instituted guidelines for routine infection prevention measures after the worldwide spread of the SARS-CoV-2 virus. Healthcare workers have been recommended to wear surgical masks and perform regular hand washing when attending low-risk individuals to safeguard against contamination. Infection prevention and control practices, which incorporate acceptable use of engineering controls (negative pressure rooms), administrative controls and PPE should be adhered to in high-risk areas [7].

The CDC has recommended airborne, and droplet infection precautions to attenuate the danger of transmissibility and cross-infection, which incorporates the mandatory use of PPE (Table 3) [7-9] including gowns, gloves, face masks and either N95, P100, or FFP2 respirators with a face shield/ googles or powered air-purifying respirator [7, 10]. Clinically suspected/confirmed COVID-19 patient should wear a cloth covering their nose and mouth, and surgical masks should preferably be reserved for healthcare workers. Frequent surface decontamination, avoiding self-contamination and enforced hand hygiene must be carefully followed [5, 7]. Providers must practice meticulous hand hygiene and disinfecting personal items (stethoscopes, phones, keys, ID tags, laptops, dictation devices, etc.). It is recommended for sanitation service workers to increase the frequency of disinfection of foremost contaminated and regularly touched surfaces like elevator buttons, door handles, light switches, grab rails, etc. [6, 11]. Majority of the orthopaedic procedures are significant aerosol-generating procedures (AGPs) 
Fig. 4 Distribution pattern of country of origin (of the first author) $(n=375)$

\section{COUNTRY OF ORIGIN}

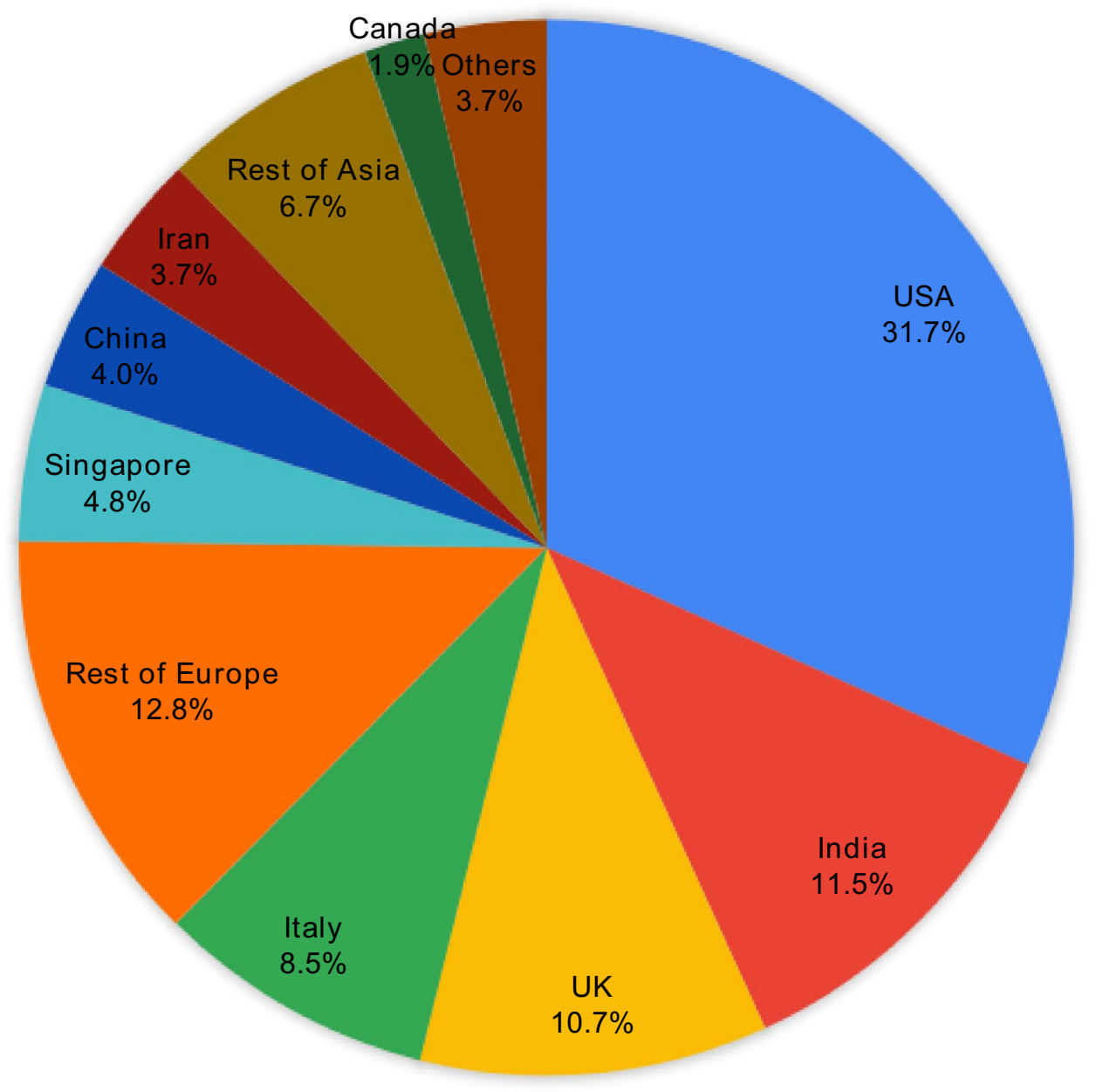

which potentially increases the risk of transmission. During high-risk AGPs (viz. use of power drills, reamers, saws, burrs, pulsed lavage) or long duration moderate-risk AGPs (viz suctioning, use of ultrasonically activated devices, osteotomes, nibblers, Gigli saw and arthroscopic/endoscopic procedures) respiratory protection above $95 \%$ is highly recommended [12]. N99/FFP3 respirators provide higher minimum filtration efficiency $(99 \%)$ to aerosolized particles compared to the $94-95 \%$ by N95/FFP2 respirators [6]. Fitted goggles are recommended as face shields are insufficient for eye protection during high-risk AGPs, followed by the first layer of surgical gloves, level 4 (water-impermeable) gown with a hood covering the neck, knee-high shoe covers and last, the outer gloves. For long-duration AGPs, the use of powered air-purifying respirators can provide an even higher level of protection and comfort. However, high costs and limited availability restricts its use. Level 2 PPE which includes N95/FFP2 respirators, fitted eye protection, AAMI level 3 or 4 gowns with hood covering neck and double gloves are recommended for a procedure on COVID-19 negative patient depending on the local prevalence of the disease and possibility of false-negative [7]. Sterile procedures in the operating room advocate the use of these PPE in conjunction with a second sterile surgical gown. Low-risk AGPs (arthrocentesis of tense swellings, skeletal traction) and bedside care require level 1 PPE including a surgical mask, gloves with face shield or goggles. When there is a probable event of fluid or droplet spread, an AAMI level 2 or 1 gown is suggested $[11,12]$.

\section{Outpatient Services}

Outpatient (OPD) services need streamlining as the patient turnover is very high and can become a potential source for exposure to coronavirus. Overcrowding has to be prevented in OPD areas. "Pre OPD clinic" is a new concept where a triage of patients is done using telemedicine and selected 
Table 3 The Recommended Use of PPE by CDC for Different Activities at Various Settings Managing Suspected/Confirmed COVID-19 Patients ( Adapted from Awad et al. [7], CDC [8], WHO [9])

\begin{tabular}{|c|c|c|c|}
\hline Sl. no & Setting & Activity & Recommended PPE \\
\hline 1 & Triage & Screening and initial examination of patients & $\begin{array}{l}\text { Surgical mask } \\
\text { Regular AAMI level-II gown } \\
\text { Goggles or face shields } \\
\text { Gloves } \\
\text { Hand hygiene before and after examining each patient }\end{array}$ \\
\hline 2 & ED consultation rooms & $\begin{array}{l}\text { Examination of patient: negative COVID-19 with no } \\
\text { signs of respiratory illness }\end{array}$ & $\begin{array}{l}\text { Surgical mask } \\
\text { Gloves } \\
\text { Regular disinfection of personal items, instruments, } \\
\text { etc. } \\
\text { Hand hygiene before and after examining each patient }\end{array}$ \\
\hline 3 & ED consultation rooms & $\begin{array}{l}\text { Examination of patient: suspected/confirmed COVID- } \\
19 \text { or patient with fever or signs of respiratory } \\
\text { illness }\end{array}$ & $\begin{array}{l}\text { Fitted, NIOSH-certified N-95 mask (if available) or sur- } \\
\text { gical mask (consider double/multiple mask technique } \\
\text { and ensure tight-fitting) } \\
\text { Regular AAMI level-II gown } \\
\text { Eye protection; goggles (covered sides of eyes) or full- } \\
\text { face shields } \\
\text { Gloves (double gloves should be considered in trauma } \\
\text { level I cases) } \\
\text { Hand hygiene before and after examining each patient }\end{array}$ \\
\hline 4 & Procedure rooms & $\begin{array}{l}\text { Providing noninvasive medical care for suspected/ } \\
\text { confirmed COVID-19 patients }\end{array}$ & $\begin{array}{l}\text { PAPR (if available) or, fitted NIOSH-certified N-95 } \\
\text { mask } \\
\text { Eye protection; goggles (covered sides of eyes) or face } \\
\text { shields } \\
\text { Disposable AAMI level-III surgical gown or coveralls } \\
\text { Double high-cuffed surgical gloves } \\
\text { Shoes worn should be fluid-resistant and easily to be } \\
\text { decontaminated } \\
\text { Hand hygiene before and after donning/doffing PPE } \\
\text { A shower after respiratory aerosol-generating proce- } \\
\text { dures is very prudent }\end{array}$ \\
\hline 5 & Operating room & $\begin{array}{l}\text { Providing emergent surgical treatment for suspected/ } \\
\text { confirmed COVID-19 patient }\end{array}$ & $\begin{array}{l}\text { PAPR is preferred for long operations or, fitted NIOSH- } \\
\text { certified N-95 mask } \\
\text { Eye protection; goggles (covered sides of eyes) or } \\
\text { face shields } \\
\text { Disposable AAMI level-III surgical gown or coveralls } \\
\text { Double high-cuffed surgical gloves } \\
\text { Shoes worn should be fluid-resistant and easily to be } \\
\text { decontaminated } \\
\text { Hand hygiene before and after donning/doffing PPE }\end{array}$ \\
\hline 6 & Operating room & $\begin{array}{l}\text { Performing aerosolized-blood generating procedures } \\
\text { for suspected/confirmed COVID-19 patient }\end{array}$ & $\begin{array}{l}\text { PAPR is preferred for long operations or, fitted NIOSH- } \\
\text { certified N-95 mask } \\
\text { Eye protection; goggles (covered sides of eyes) or } \\
\text { face shields } \\
\text { Surgical hood with ties (head and neck covering) } \\
\text { Disposable AAMI level-III surgical gown or coveralls } \\
\text { Double high-cuffed surgical gloves } \\
\text { Shoes worn should be fluid-resistant and easily to be } \\
\text { decontaminated } \\
\text { Hand hygiene before and after donning/doffing PPE } \\
\text { A shower after an aerosolized-blood generating proce- } \\
\text { dure is very prudent }\end{array}$ \\
\hline 7 & Recovery room & PPE doffing & $\begin{array}{l}\text { Special attention is warranted to avoid self-contamina- } \\
\text { tion during PPE doffing }\end{array}$ \\
\hline
\end{tabular}

AAMI Association of the Advancement of Medical Instrumentation, EDemergency department, NIOSHNational Institute for Occupational Safety and Health, PAPR powered air-purifying respirator

${ }^{a}$ Alternately, vertical strips of tape can keep gloves secured to the gown

${ }^{\mathrm{b}}$ Disposable shoe covers might increase risk of contamination 
patients who require a physical examination by experts are given an appointment for hospital visits. The rest of the patients can be treated with the help of teleconsultations or telephonic advice [13]. Specific measures like frequent announcements, pictorial signage and individual verbal communication with a dedicated team for guidance are required for illiterate persons without access to electronic media [14].

Screening of patients should be done at the OPD entrance where history related to COVID-19 exposure, cough, fever and shortness of breath should be enquired, and temperature and oxygen saturation recorded. Patients should be directed to respective departments to avoid unnecessary movement within hospital premises. Mouth and nostrils of patients should be covered at all times and entry denied without the same. Patients should be instructed to avoid physical contact with railing, furniture and door handles and to strictly follow hand hygiene. Consultation room should be spacious with proper ventilation with a separate entry and exit point for patients. Strict protocols for restricting the number of accompanying persons entering are required $[15,16]$.

Rooms dedicated for cast application, wound dressings and injections have to be periodically cleaned with sodium hypochlorite $1 \%$ every day. Disposable sterile sheets and gowns should be used in these areas and discarded properly after usage. Staff in charge of these services should ensure that proper protocol is followed to ensure both sterility and prevention of the spread of coronavirus. Patients requiring elective surgery should be counselled and reassured about the delay in reopening elective theatres. Procedures to alleviate pain and discomfort of such patients can be carried out in daycare services. Interdepartmental services from physiotherapy and referrals have to be carried out with proper coordination and dedicated time slots. One must use a single most reliable radiological investigation most likely to give a definitive diagnosis. Radiographs must be done only if they affect the outcome of treatment [17].

In certain centres of Europe and USA during the surge of COVID-19 cases, self-sufficient sub-teams were established in separate areas to reduce the burden on emergency departments of tertiary centres, and freeing personnel and resources to combat the pandemic. Such sub-teams provide emergency and outpatient services to orthopaedic patients while the rest are shielded from the pandemic. These dedicated centres could potentially serve as a model for other surgical and medical subspecialty clinics both during and after the COVID-19 pandemic [18].

\section{Elective Orthopaedic Surgery}

As a measure to protect patients from infection and for rationalizing resources, elective and non-emergency surgeries have been postponed at most centres during the pandemic surge. These postponed elective procedures are required to reduce pain, improve function and quality of life and prevent complications or disease progression. The number of suffering patients has reached critical volumes that will need to be managed sensibly once the COVID-19 pandemic improves [19].

Each hospital and health system should evaluate the situation in terms of COVID-19 infection prevalence, healthcare personnel, PPE supply and state policy permissions when determining how and when to start elective procedures. According to guidelines defined by members of American Association of Hip and Knee Surgeons [11], elective surgery may be resumed when lockdown in the region has been lifted and a mandate allowing the return to elective surgery has been issued by regional authorities or when the number of COVID-19 cases in the region has been consistently declining. The surgical facility should admit COVID-19 negative patients in a designated COVID free area separate from COVID-19 suspect/positive patients; the facility should also have an adequate supply of PPE, testing kits and should be able to perform surgery safely with low risk of transmission of the virus. The facility should be able to maintain social distancing throughout the process in all phases (preoperatively, intraoperatively and postoperatively).

Before elective surgeries, screening of patients should be done. Patients with COVID positive reports should not undergo elective procedures. Contacts of positive cases should only be treated after quarantine and isolation protocols are completed. Postoperatively hospital stay can be minimized with the help of telemedicine and telerehabilitation [11, 20, 21].

It is anticipated that demands for elective surgery shall increase after the resumption of services; therefore, it is mandatory to prioritize patients, depending on discomfort and pain. Several guidelines for conducting orthopaedic procedures during the COVID-19 have been laid down by various organizations, but most put the onus of decision-making on individual hospital systems as well as surgeons [20,21]. Eventually, surgeons must weigh the benefits of performing surgery with the potential impact on public health services.

\section{Emergency Orthopaedic Surgery}

Emergency trauma surgeries cannot be postponed nor delayed beyond a reasonable duration. In addition, patients with a fracture may be at a higher risk of COVID-19 pneumonia [22]. The basic principle to follow in this pandemic is to limit hospital stay while maintaining a high standard of care. Therefore, the timing of treatment plays a critical role in this scenario. Here, we have divided orthopaedic emergencies into two subheadings- "emergency-within $24 \mathrm{~h}$ " and "urgent—between 24 and 48 h". Emergency priority 
cases are those in which if not surgically intervened immediately, they may result in loss of life or limb or paralysis [6]. Urgent cases are those which need to be done soon when medically stable (Table 4)[6, 23-25].

All patients who need emergency surgery need to complete COVID-19 swab test prior to surgery. Patients should be placed in the transitional area while waiting for results [26]. Once the result is obtained, the patient can be shifted to the main orthopaedic ward for further care and management. If COVID-19 testing is not possible, then a 'Human immunodeficiency virus HIV model', as suggested by Jain et al. [27], can be adopted in the operative management of patients requiring emergency procedures and such patients should be treated as COVID-19 positive until proved otherwise.

\section{Conservative Fracture Management}

COVID-19 pandemic has shifted the focus to conservative management for treatment of several fractures. Conservative or a non-surgical therapeutic approach may provide an alternative in non-obligatory fractures in the current COVID-19 pandemic and perhaps later on as well. Traditional concepts of closed reduction may still hold good especially in undisplaced or minimally displaced fractures of upper and lower limb. Supportive treatment with skeletal traction can be used for multiple fractures especially in the lower limb. Geriatric patients with stable proximal femoral fractures can be managed conservatively with the use of splints or skeletal traction, especially those who are not surgically fit [24]. Long knee brace and splint can be used for acute knee injuries. Removable splints for upper limb and lower limb should be advised to prevent multiple visits and consultations. British Orthopaedic Association states that plaster cutting is not considered as an AGP as it uses an oscillating device and when used correctly it will not cause any soft tissue injury to generate aerosols [28]. Survival of COVID-19 virus on cast material has not been confirmed in current literature [29]. The cast saw generates fine dust so an attached vacuum is recommended, and cast shears should be considered as an alternative [28]. These techniques should be used by a trained professional with proper understanding of casting principles. Removable splints, non-circumferential cast or soft cast that can be removed with scissors easily at home should be recommended. When home management is advised, the caregiver should be explicitly explained about the correct technique of cast removal. If a patient on cast gets infected with COVID-19 or is self-isolating due to contact history, the patient should enquire whether he/she can come to the hospital facility or get treated by teleconsultation. If required, cast removal can be delayed till the symptoms subside or self-isolation is completed. Patients should be advised about the signs and symptoms of complications such as pressure sores, numbness and swelling and also provided medical attention at the earliest [28].

\section{Rehabilitation}

Rehabilitation is an integral part of orthopaedic care which is severely affected during the pandemic. Telerehabilitation is the new face of rehabilitation services in the current scenario [30]. It is an efficient method to connect remote patients with specialists and significantly curtails unnecessary hospital visits. Monitoring of progress by accessing daily activities and motor functions can be done conveniently. Evidence show home-based rehabilitation is effective in orthopaedic patients suffering from common musculoskeletal problems like paralysis, muscular dystrophy, spasticity, bladder incontinence and also severe debilitating deformities [31, 32]. For elderly patients with fragility fractures, telerehabilitation can improve mobility and independence, which can protect them from deteriorating health, bedsores and chest infections [33]. The major drawback of telerehabilitation is the scarcity of equipment in remote areas and unawareness among local population.

\section{Specific Orthopaedic Subspecialities}

\section{Fragility Fractures}

Fragility fractures are those fractures that result from forces that would otherwise not result in a fracture, known as 'lowenergy' trauma such as equivalent to a fall from standing height or lesser. Fragility hip fractures are usually sustained in elderly people who have associated co-morbidities. COVID-19 pandemic has reduced the numbers of highenergy trauma like road traffic accidents, but low-energy injuries like peritrochanteric and spine fractures are still prevalent. These hip and spine fractures are associated with increased mortality, reduction in quality of life and functional limitations along with substantial economic burden to the health sector, further compromising COVID-19 treatment [34]. Therefore, a well-planned mitigation strategy, that includes multidisciplinary teamwork including rehabilitation and telemedicine follow-up is necessary in this COVID era. There is a likely connection between COVID19 infection and fragility hip fracture in elderly patients. Fatigue and weakness caused by COVID-19 disease can lead to fragility fracture. Therefore, COVID-19 infection should be considered in elderly patients with fragility hip fracture during the coronavirus pandemic [35].

Obligatory fractures such as hip and vertebral fractures with neurological deficit require operative intervention. The British Geriatric Society recommends that elderly patient 


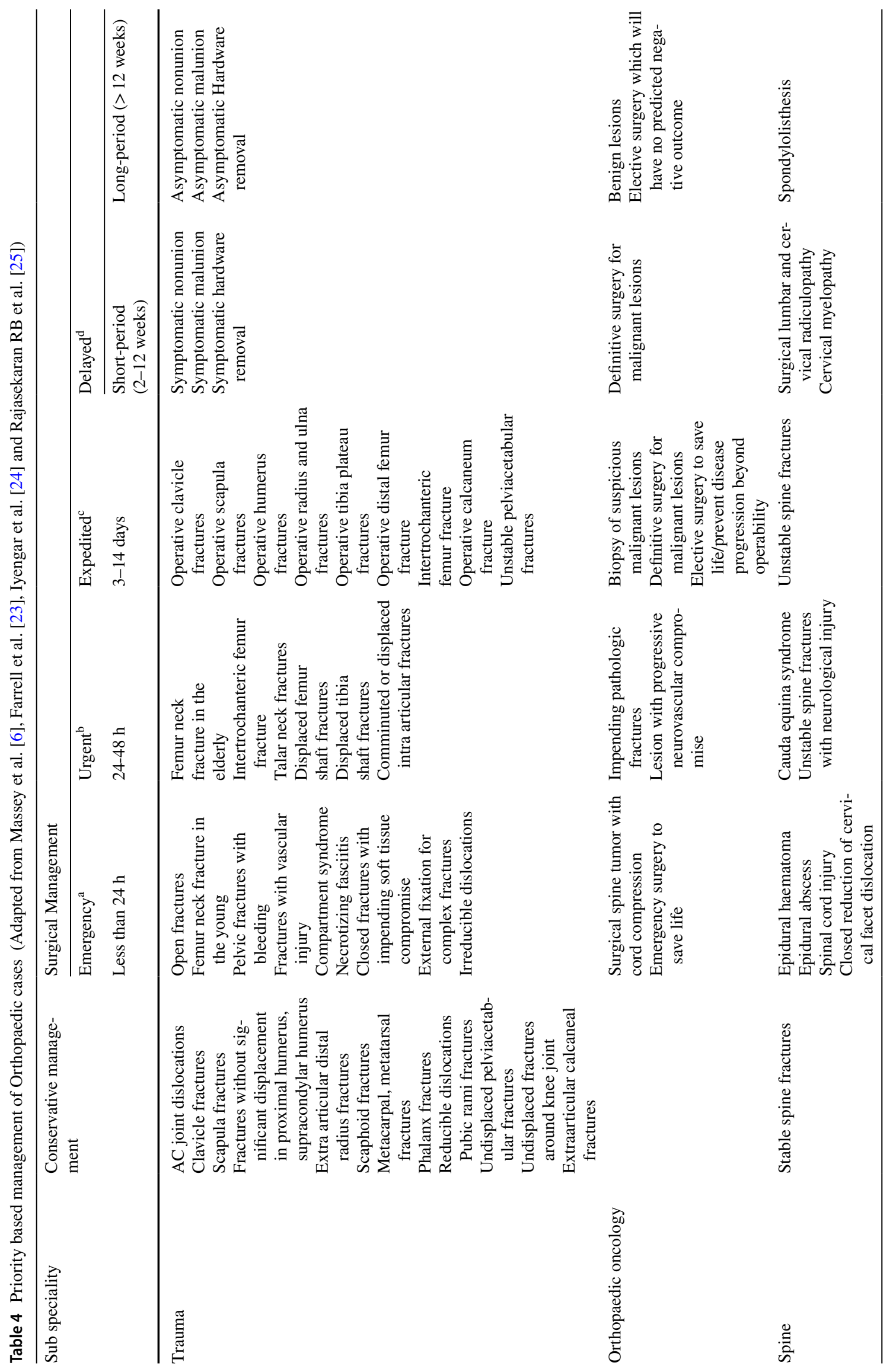




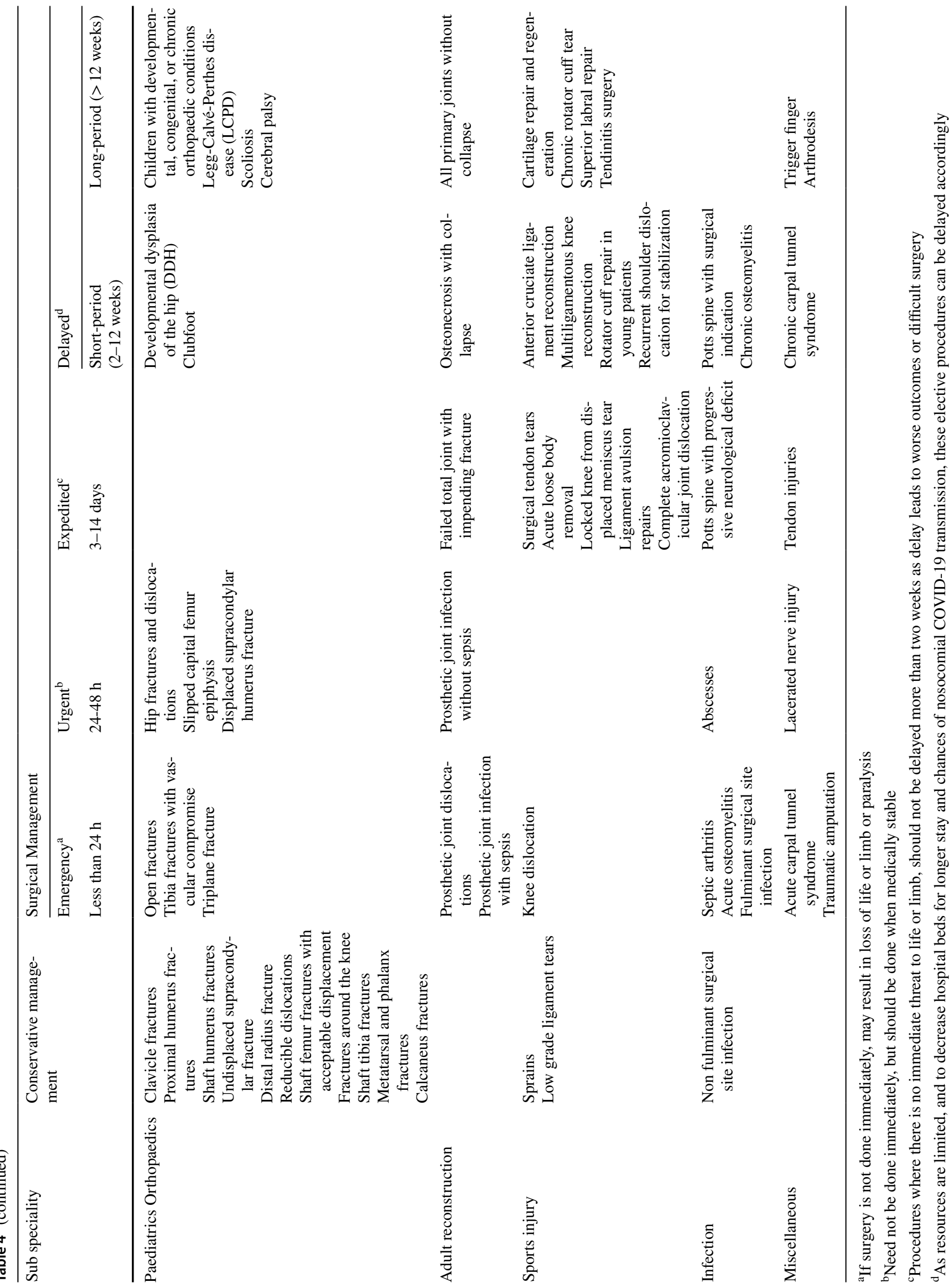


with hip fractures should undergo minimal invasive procedure which require less operating time, minimizes blood loss, allows immediate post-operative mobilisation to allow rehabilitation and reduced length of stay with a primary aim to reduce exposure to COVID-19 infection [36]. Fragility hip fractures can be treated by hemi-replacement arthroplasties for intracapsular fractures and intra or extramedullary implants for peritrochanteric fractures. In addition, hemiarthroplasty can enable immediate mobilization and negate the risk of non-union in these cases. Intramedullary nails are load-bearing and reduce the bone-implant interface [37]. There is a higher mortality rate in patients with a hip fracture in the elderly and an associated positive test for COVID-19 [38]. COVID-19 patients with high-risk comorbidities can be managed conservatively on traction immobilization.

Non-obligatory fractures like distal radius fracture, vertebral fracture without neurological deficit or proximal humerus fracture should be managed conservatively, as much as possible. The conservative treatment of an undisplaced vertebral fracture includes analgesia with a period of bed rest and later mobilisation with thoracolumbar support. Fracture of distal radius if displaced should be reduced and maintained in removable splint or slab. This allows selfremoval of splints or plasters at home and avoids an outpatient visit. Similarly, most of the fractures of the proximal humerus can be managed with an arm pouch sling. Followup of these patients can be done through telemedicine facility whenever possible [37].

\section{Orthopaedic Oncology}

Elective surgeries were cancelled to mitigate disease transmission, but essential services like cancer management needed to continue to prevent disease progression and reduce mortality [39, 40]. A reorganisation of the main arms viz. triage, multidisciplinary team (MDT), clinic, surgery and adjuvant therapies, is vital to deliver effective cancer care continuously throughout the peak of the pandemic. Bone tumours and soft tissue sarcomas have a very low incidence and amount to less than $1 \%$ of all cancers [41]. The Oxford Bone Tumour and Soft Tissue Sarcoma service suggested virtual MDT meetings, teleconsultations for follow-up patients, surgery only to save life/prevent disease progression beyond operability, use of COVID free hospital for cancer surgery and purposeful postponement of adjuvant therapies for stable cancers, for mitigating risks of the pandemic [25]. Though telemedicine is valuable for the care of cancer patients, lack of physical examination and delay in diagnosis of metastasis are hard limitations [42].

The guidelines of other Orthopaedic subspecialties are discussed in Table 4.

\section{Orthopaedic Resident Teaching}

COVID-19 has severely affected the teaching and training in school of medicine. Most of the institutions have suspended classroom teaching and various examinations are cancelled or postponed. Teaching is conducted via webinars, online modes, or e-learning, leading to less face-toface interaction, negligible feedback from the scholars and lack of accreditation, requiring intense self-discipline. . Training in clinical and surgical skills of the residents is jeopardized with only limited exposures during a simulation-based setup which is demanding and less reproducible in clinical practice with limited availability at several medical schools [43]. The most important objective of orthopaedic teaching and examination during the COVID19 pandemic is to prevent infection to the residents, examiners and patients. The maintenance of social distancing norms, PPE and hand hygiene is mandatory [44, 45].

Virtual case presentations for regular clinical teaching as well as final professional exit exams require a collection of clinical images which can be made to replicate a reallife clinical scenario. The representative clinical case scenario should have a concise clinical history with relevant questions of the disease in such a way that can give clues to reach the proper diagnosis in an examination setting [45]. The viva can further be extended by asking questions about additional history and examination findings.

The use of an interactive computer touch screen during virtual examination can help the examiner to ask students to draw clinically relevant anatomical angles or to demonstrate pertinent clinical findings (viz. three-point bony landmarks at the elbow, Bryant's triangle for supratrochanteric shortening, etc.) and to identify relevant findings on radiographs. The final qualifying examination is always stressful irrespective of examination pattern. This newer virtual examination adds more anxiety to the candidates. This anxiety can be mitigated by prior orientation, mock tests and demonstration of representative cases on the examination website portal which will help both the examiner and candidate to conform to the new pattern and formally prepare for the virtual practical examination [44-46].

The major difficulties in online teaching are digital illiteracy, inadequate internet connectivity, access to good audiovisual devices and the privacy policy of teleconferencing software which may be solved using an able Information Technology department. The flooding of webinars and online resources is itself an epidemic of e-learning but this online training and education are here to remain even in the post-COVID-19 era. The orthopaedic surgeon should proficiently use cutting-edge technology, artificial intelligence for teaching graduate and post-graduate 
students and maintain a positive attitude during this point and overcome these challenges by motivating younger individuals [43, 47].

\section{Telemedicine and Virtual Conferencing}

Telemedicine in orthopaedics broadly refers to virtual visit interactions, which are synchronous outpatient video interactions between patients and healthcare providers. Orthopaedic assessment relies on clinical examination, which makes it necessary to have an accurate and validated patient examination method using telemedicine. Tachakra et al. demonstrated that telemedicine has good accuracy of examination and reported no significant differences in adverse events or reduced outcomes in patients using telemedicine [48]. It is important to prepare and standardize the conditions of the virtual physical examination that can help to optimize the quality and efficiency of the telemedicine consultation. Patients can be sent a pre-consultation checklist to analyse their chief concern, along with diagrams to localise pain, deformity, swelling and/or sensory abnormalities. Specific tools that assist virtual physical examination like web-based goniometers are compatible with most telemedicine applications.

Tele-Orthopaedics has the most effective utilization in joint arthroplasty, general postoperative care and fracture management. Its most promising benefits include high patient satisfaction rates with increased patient convenience and access while decreased overhead costs for providers. Challenges related to telemedicine include lack of awareness, access and technology literacy in both providers and patients [49]. Implementing and maintaining telemedicine capabilities for healthcare systems requires considerable investment. The increased medicolegal liability from making diagnoses, recommending surgery and following up without the ability to perform a physical examination may deter adoption. By addressing the challenges in implementation and training, raising awareness and improving education, streamlining technology and collaborating with patients, providers and payors to align incentives, telemedicine can prove beneficial to the sector of orthopaedic surgery $[43,47]$.

\section{Conclusion}

COVID-19 has had a significant impact on all aspects of orthopaedic care and practice. The pandemic has affected outpatient clinics, emergency and elective surgery, rehabilitation, resident training, personnel management, use of PPE, telemedicine and all sub-specialities of orthopaedics.
Orthopaedic practice will require the incorporation of new technologies, restructuring of health systems and reorganizing of training programs for optimal patient care. There would also be a need for frequent review of emerging literature to provide evidence-based guidelines to the orthopaedic fraternity which will not only help in mitigation of transmission of disease but also ensure continuity of optimum patient care.

Supplementary Information The online version contains supplementary material available at https://doi.org/10.1007/s43465-021-00354-0.

\section{Compliance with ethical standards}

Conflict of interest On behalf of all authors, the corresponding author states that there is no conflict of interest.

Ethical standard This article does not contain any studies with human or animal subjects performed by the any of the authors.

Informed consent For this type of study informed consent is not required.

\section{References}

1. Arksey, H., \& O’Malley, L. (2005). Scoping studies: towards a methodological framework. International Journal of Social Research Methodology, 8, 19-32. https://doi.org/10.1080/13645 57032000119616.

2. Moher, D., Liberati, A., Tetzlaff, J., Altman, D. G., \& PRISMA Group. (2009). Preferred reporting items for systematic reviews and meta-analyses: the PRISMA statement. BMJ, 339, 2535. https://doi.org/10.1136/bmj.b2535.

3. Tricco, A. C., Antony, J., Zarin, W., Strifler, L., Ghassemi, M., Ivory, J., et al. (2015). A scoping review of rapid review methods. BMC Med., 13, 224. https://doi.org/10.1186/s12916-015-0465-6.

4. Kidd, V. D. (2020). Redeployment of orthopaedic advanced practice providers at academic medical centers during the COVID-19 pandemic. Orthopaedic Nursing, 39, 215-217. https://doi.org/10. 1097/NOR.0000000000000689.

5. O'Connor, M., Nieuwoudt, L., \& Marais, L. C. (2020). Orthopaedics and COVID-19: The surgery, the surgeon and the susceptible-a scoping review. SA Orthopaedic Journal Internet., 19, 129-137. https://doi.org/10.17159/2309-8309/2020/v19n3a1.

6. Massey, P. A., McClary, K., Zhang, A. S., Savoie, F. H., \& Barton, R. S. (2020). Orthopaedic surgical selection and inpatient paradigms during the coronavirus (COVID-19) pandemic. Journal of American Academy of Orthopaedic Surgeons, 28, 436-450. https://doi.org/10.5435/JAAOS-D-20-00360.

7. Awad, M. E., Rumley, J. C. L., Vazquez, J. A., \& Devine, J. G. (2020). Perioperative considerations in urgent surgical care of suspected and confirmed COVID-19 orthopaedic patients: operating room protocols and recommendations in the current COVID-19 pandemic. Journal of American Academy of Orthopaedic Surgeons, 28, 451-463. https://doi.org/10.5435/JAAOS-D-20-00227.

8. Centers for Disease Control and Prevention. (2020). Infection control guidance for healthcare professionals about coronavirus (COVID-19). https://www.cdc.gov/coronavirus/2019-ncov/hcp/ infection-control.html. Accessed 8th Nov 2020. 
9. World Health Organization. (2020). Rational use of personal protective equipment for coronavirus disease (COVID-19) and considerations during severe shortages: interim guidance, 6 April 2020. World Health Organization; 2020. https://www.who. int/publications/i/item/rational-use-of-personal-protective-equip ment-for-coronavirus-disease-(covid-19)-and-considerations-during-severe-shortages. Accessed 8 Nov 2020.

10. Verma, V., Nagar, M., Jain, V., Santoshi, J. A., Dwivedi, M., Behera, P., et al. (2020). Adapting Policy Guidelines For Spine Surgeries During COVID-19 pandemic in view of evolving evidences: An early experience from a tertiary care teaching hospital. Cureus., 12, e9147. https://doi.org/10.7759/cureus.9147.

11. Parvizi, J., Gehrke, T., Krueger, C. A., Chisari, E., Citak, M., Van Onsem, S., et al. (2020). Resuming elective orthopaedic surgery during the COVID-19 pandemic: Guidelines developed by the international consensus group (ICM). Journal of Bone and Joint Surgery, 102, 1205-1212. https://doi.org/10.2106/JBJS.20.00844.

12. Geevarughese, N. M., \& Ul-Haq, R. (2020). Aerosol generating procedures in orthopaedics and recommended protective gear. Journal of Clinical Orthopaedics and Trauma. https://doi.org/ 10.1016/j.jcot.2020.08.019.

13. Abolghasemian, M., Ebrahimzadeh, M. H., Enayatollahi, M., Honarmand, K., Kachooei, A. R., Mehdipoor, S., et al. (2020). Iranian Orthopedic Association (IOA) Response Guidance to COVID-19 pandemic april 2020. The Archives of Bone and Joint Surgery, 8(Suppl 1), 209-217. https://doi.org/10.22038/ABJS. 2020.47678.2370.

14. Lal, H., Sharma, D. K., Patralekh, M. K., Jain, V. K., \& Maini, L. (2020). Out Patient Department practices in orthopaedics amidst COVID-19: The evolving model. Journal of Clinical Orthopaedics and Trauma, 11, 700-712. https://doi.org/10.1016/j.jcot. 2020.05.009.

15. Chang Liang, Z., Wang, W., Murphy, D., \& Po Hui, J. H. (2020). Novel coronavirus and orthopaedic surgery: Early experiences from Singapore. Journal of Bone and Joint Surgery. American Volume, 102, 745-749. https://doi.org/10.2106/JBJS.20.00236.

16. Athey, A. G., Cao, L., Okazaki, K., Zagra, L., Castelli, C. C., Kendoff, D. O., et al. (2020). Survey of AAHKS international members on the impact of COVID-19 on hip and knee arthroplasty practices. Journal of Arthroplasty, 35, S89-S94. https:// doi.org/10.1016/j.arth.2020.04.053.

17. Rossi, B., Zoccali, C., Baldi, J., di Uccio, A. S., Biagini, R., De Luca, A., et al. (2020). Reorganization tips from a sarcoma unit at time of the COVID-19 pandemic in Italy: Early experience from a Regional Referral Oncologic Center. Journal of Clinical Medicine., 9, 1868. https://doi.org/10.3390/jcm9061868.

18. MacKechnie, M. C., Nadeau, M., Deering, E., Thaller, J., \& MacKechnie, M. A. (2020). Orthopaedic walk-in clinics: A model to lessen the burden on Emergency Departments during the COVID-19 pandemic. Journal of Orthopaedics, 20, 293-296. https://doi.org/10.1016/j.jor.2020.05.014.

19. Ding, B. T. K., Tan, K. G., Oh, J. Y., \& Lee, K. T. (2020). Orthopaedic surgery after COVID-19-a blueprint for resuming elective surgery after a pandemic. International Journal of Surgery, 80, 162-167. https://doi.org/10.1016/j.ijsu.2020.07.012.

20. Kort, N. P., Zagra, L., Barrena, E. G., Tandogan, R. N., Thaler, M., Berstock, J. R., \& Karachalios, T. (2020). Resuming hip and knee arthroplasty after COVID-19: Ethical implications for wellbeing, safety and the economy. Hip International, 30, 492-499. https:// doi.org/10.1177/1120700020941232.

21. Sarac, N. J., Sarac, B. A., Schoenbrunner, A. R., Janis, J. E., Harrison, R. K., Phieffer, L. S., et al. (2020). A review of state guidelines for elective orthopaedic procedures during the COVID-19 Outbreak. Journal of Bone and Joint Surgery. American Volume, 102, 942-945. https://doi.org/10.2106/JBJS. 20.00510 .
22. Mi, B., Chen, L., Xiong, Y., Xue, H., Zhou, W., \& Liu, G. (2020). Characteristics and early prognosis of COVID-19 infection in fracture patients. Journal of Bone and Joint Surgery. American Volume, 102, 750-758. https://doi.org/10.2106/JBJS.20.00390.

23. Farrell, S., Schaeffer, E. K., \& Mulpuri, K. (2020). Recommendations for the care of pediatric orthopaedic patients during the COVID-19 pandemic. Journal of American Academy of Orthopaedic Surgeons, 28, e477-e486. https://doi.org/10.5435/ JAAOS-D-20-00391.

24. Iyengar, K., Vaish, A., \& Vaishya, R. (2020). Revisiting conservative orthopaedic management of fractures during COVID19 pandemic. Journal of Clinical Orthopaedics and Trauma, 11, 718-720. https://doi.org/10.1016/j.jcot.2020.05.010.

25. Rajasekaran, R. B., Whitwell, D., Cosker, T. D. A., \& Gibbons, C. L. M. H. (2020). Service delivery during the COVID-19 pandemic: Experience from The Oxford Bone Tumour and Soft Tissue Sarcoma service. Journal of Clinical Orthopaedics and Trauma, 11(Suppl 4), S419-S422. https://doi.org/10.1016/j.jcot. 2020.05.035.

26. National Clinical Programme in Surgery. (2020). COVID-19 Guidelines: Intraoperative recommendations when operating on suspected COVID infected patients. https://www.rcsi.com/surge ry/coronavirus/surgical-practice/clinical-guidance-for-surgeons. Accessed 8 Nov 2020.

27. Jain, V. K., \& Vaishya, R. (2020). COVID-19 and orthopaedic surgeons: The Indian scenario. Tropical Doctor, 50, 108-110. https://doi.org/10.1177/0049475520921616.

28. British Orthopaedic Association. (2020). British Orthopaedic Association casting committee guidance for casting practice in the current COVID-19 pandemic. https://www.boa.ac.uk/resources/ guidance-for-casting-practice-in-the-current-covid-19-pandemic. html. Accessed 8 Nov 2020.

29. van Doremalen, N., Bushmaker, T., Morris, D. H., Holbrook, M. G., Gamble, A., Williamson, B. N., et al. (2020). Aerosol and Surface Stability of SARS-CoV-2 as Compared with SARS-CoV-1. New England Journal of Medicine, 382, 1564-1567. https://doi. org/10.1056/NEJMc2004973.

30. Leochico, C. F. (2020). Adoption of telerehabilitation in a developing country before and during the COVID-19 pandemic. Annals of Physical and Rehabilitation Medicine, 63, 563-564. https://doi. org/10.1016/j.rehab.2020.06.001.

31. Azhari, A., \& Parsa, A. (2020). Covid-19 outbreak highlights: Importance of home-based rehabilitation in orthopedic surgery. The Archives of Bone and Joint Surgery, 8(Suppl1), 317-318. https://doi.org/10.22038/abjs.2020.47777.2350.

32. Li, D., Yang, Z., Kang, P., \& Xie, X. (2017). Home-based compared with hospital-based rehabilitation program for patients undergoing total knee arthroplasty for osteoarthritis: A systematic review and meta-analysis of randomized controlled trials. American Journal of Physical Medicine and Rehabilitation, 96, 440-447. https://doi.org/10.1097/PHM.0000000000000621.

33. Catellani, F., Coscione, A., D’Ambrosi, R., Usai, L., Roscitano, C., \& Fiorentino, G. (2020). Treatment of proximal femoral fragility fractures in patients with COVID-19 during the SARS-CoV-2 outbreak in Northern Italy. Journal of Bone and Joint Surgery. American Volume, 102, e58. https://doi.org/10.2106/JBJS.20. 00617.

34. Cauley, J. A. (2013). Public health impact of osteoporosis. Journals of Gerontology. Series A, Biological Sciences and Medical Sciences, 68, 1243-1251. https://doi.org/10.1093/gerona/glt093.

35. Shariyate, M. J., \& Kachooei, A. R. (2020). Association of new coronavirus disease with fragility hip and lower limb fractures in elderly patients. The Archives of Bone and Joint Surgery, 8(Suppl1), 297-301. https://doi.org/10.22038/abjs.2020.47626. 2333. 
36. British Geriatrics Society. Managing Hip Fractures during COVID19. (2020). https://www.bgs.org.uk/blog/managing-hipfractures-during-covid19. Accessed 8 Nov 2020.

37. Upadhyaya, G. K., Iyengar, K., Jain, V. K., \& Vaishya, R. (2020). Challenges and strategies in management of osteoporosis and fragility fracture care during COVID-19 pandemic. Journal of Orthopaedics, 21, 287-290. https://doi.org/10.1016/j.jor.2020.06. 001.

38. Muñoz Vives, J. M., Jornet-Gibert, M., Cámara-Cabrera, J., Esteban, P. L., Brunet, L., Delgado-Flores, L., et al. (2020). mortality rates of patients with proximal femoral fracture in a worldwide pandemic: Preliminary results of the Spanish HIP-COVID Observational Study. The Journal of Bone and Joint Surgery, 102, e69. https://doi.org/10.2106/JBJS.20.00686.

39. Saini, K. S., de Las, H. B., de Castro, J., Venkitaraman, R., PoeIman, M., Srinivasan, G., et al. (2020). Effect of the COVID-19 pandemic on cancer treatment and research. The Lancet Haematology, 7, e432-e435. https://doi.org/10.1016/S2352-3026(20) 30123-X.

40. Vrdoljak, E., Sullivan, R., \& Lawler, M. (2020). Cancer and coronavirus disease 2019; how do we manage cancer optimally through a public health crisis? European Journal of Cancer, 132, 98-99. https://doi.org/10.1016/j.ejca.2020.04.001.

41. Franchi, A. (2012). Epidemiology and classification of bone tumors. Clinical Cases in Mineral and Bone Metabolism, 9, 92-95.

42. Bluman, E. M., Fury, M. S., Ready, J. E., Hornick, J. L., \& Weaver, M. J. (2020). Orthopedic telemedicine encounter during the COVID-19 pandemic: A cautionary tale. Trauma Case Reports, 28, 100323. https://doi.org/10.1016/j.tcr.2020.100323.

43. Haider, Z., Aweid, B., Subramanian, P., \& Iranpour, F. (2020). Telemedicine in orthopaedics and its potential applications during COVID-19 and beyond: A systematic review. Journal of Telemedicine and Telecare, 2020, 1357633X20938241. https://doi. org/10.1177/1357633X20938241.
44. Malhotra, R., Gautam, D., George, J., Goyal, D., \& Ansari, M. T. (2020). Conducting orthopaedic practical examination during the Covid-19 pandemic. Journal of Clinical Orthopaedics and Trauma, 11(Suppl 4), S448-S455. https://doi.org/10.1016/j.jcot. 2020.07.002.

45. Iyengar, K. P., Jain, V. K., \& Vaishya, R. (2020). Virtual postgraduate orthopaedic practical examination: a pilot model. Postgraduate Medical Journal, 2020, 138726. https://doi.org/10.1136/ postgradmedj-2020-138726.

46. Foong, W. S., Teo, H. L. T., Wang, D. H. B., \& Loh, S. Y. J. (2020). Challenges and adaptations in training during pandemic COVID-19: Observations by an orthopedic resident in Singapore. ActaOrthopaedica, 2020, 1-5. https://doi.org/10.1080/17453674. 2020.1786641.

47. Lanham, N. S., Bockelman, K. J., \& McCriskin, B. J. (2020). Telemedicine and orthopaedic surgery: The COVID-19 pandemic and our new normal. JBJS Reviews, 8, e2000083. https://doi.org/ 10.2106/JBJS.RVW.20.00083.

48. Tachakra, S., Lynch, M., Newson, R., Stinson, A., Sivakumar, A., Hayes, J., \& Bak, J. (2000). A comparison of telemedicine with face-to-face consultations for trauma management. Journal of Telemedicine and Telecare, 6(Suppl 1), S178-S181. https://doi. org/10.1258/1357633001934591.

49. Makhni, M. C., Riew, G. J., \& Sumathipala, M. G. (2020). Telemedicine in orthopaedic surgery: Challenges and opportunities. Journal of Bone and Joint Surgery. American Volume, 102, 1109-1115. https://doi.org/10.2106/JBJS.20.00452.

Publisher's Note Springer Nature remains neutral with regard to jurisdictional claims in published maps and institutional affiliations 